\title{
Implementation of Antiretroviral Therapy for Life in Pregnant/Breastfeeding HIV+ Women (Option B+) Alongside Rollout and Changing Guidelines for ART Initiation in Rural Zimbabwe: The Lablite Project Experience
}

\author{
Deborah Ford, PhD, * Margaret Muzambi, BSc, † Misheck J. Nkhata, MSc, $\neq$ George Abongomera, MSc, $\S$ \\ Sarah Joseph, PhD,* Makosonke Ndlovu, BSc, † Travor Mabugu, MSc, † Caroline Grundy, LLB,* \\ Adrienne K. Chan, MD, $\neq \|$ Fabian Cataldo, PhD, $\neq$ Cissy Kityo, MSc, $\$$ Janet Seeley, PhD, $\rceil$ \\ Elly Katabira, FRCP,\# Charles F. Gilks, DPhil,** Andrew Reid, MRCP, $\dagger$ James Hakim, PhD, $\dagger$ and
}

Diana M. Gibb, MD, * the Lablite Team

Background: Lifelong antiretroviral therapy (ART) for pregnant and breastfeeding women (Option B+) was rolled out in Zimbabwe from 2014, with simultaneous raising of the CD4 treatment threshold to 500 cells per cubic millimeter in nonpregnant/breastfeeding adults and children 5 years and over.

Methods: Lablite is an implementation project in Zimbabwe, Malawi, and Uganda evaluating ART rollout. Routine patient-level data were collected for 6 months before and 12 months after Option B+ rollout at a district hospital and 3 primary care facilities in Zimbabwe (2 with outreach ART and 1 with no ART provision before Option $\mathrm{B}+$ ).

Results: Between September 2013 and February 2015, there were 1686 ART initiations in the 4 facilities: $91 \%$ adults and $9 \%$ children younger than 15 years. In the 3 facilities with established ART, initiations rose from 300 during 6 months before Option $\mathrm{B}+$ to 869 (2.9-fold) and 463 (1.5-fold), respectively, 0-6 months and 6-12

Received for publication July 1, 2016; accepted October 31, 2016.

From the *MRC Clinical Trials Unit at University College London, London, United Kingdom; †University of Zimbabwe, Harare, Zimbabwe; $\$$ Dignitas International, Zomba, Malawi; §Joint Clinical Research Centre, Kampala, Uganda; \#Division of Infectious Diseases, Department of Medicine, Sunnybrook Health Sciences Centre, University of Toronto, Toronto, Ontario, Canada; TMRC/UVRI Uganda Research Unit of AIDS, Entebbe, Uganda; \#Infectious Diseases Institute, Makerere University, Mulago, Uganda; and **School of Population Health, University of Queensland, Queensland, Australia.

Supported by Department for International Development, United Kingdom. Presented at International Conference on AIDS and STIs in Africa, November 30, 2015, Harare, Zimbabwe.

The authors have no conflicts of interest to disclose.

This article is an output from a project funded by DFID for the benefit of developing countries. The views expressed are not necessarily those of DFID.

D.F. and M.M. have contributed equally.

Correspondence to: Deborah Ford, $\mathrm{PhD}$, MRC Clinical Trials Unit at University College London, Aviation House, 125 Kingsway, London WC2B 6NH, United Kingdom (e-mail: deborah.ford@ucl.ac.uk).

Copyright (C) 2016 The Author(s). Wolters Kluwer Health, Inc. This is an open-access article distributed under the terms of the Creative Commons Attribution-Non Commercial License 4.0 (CCBY-NC), where it is permissible to download, share, remix, transform, and buildup the work provided it is properly cited. The work cannot be used commercially without permission from the journal. months after Option $\mathrm{B}+$. Post-Option $\mathrm{B}+$, an estimated $43 \%$ of pregnant/breastfeeding women needed ART for their own health, based on World Health Organization stage $3 / 4$ or CD $4 \leq 350$ per cubic millimeter $(64 \%$ for CD4 $\leq 500)$. Seventy-four men $(22 \%)$ and 123 nonpregnant/breastfeeding women (34\%) initiated ART with CD4 >350 after the CD4 threshold increase. Estimated 12-month retention on ART was $79 \%(69 \%-87 \%)$ in Option B+ women (significantly lower in younger women, $P=0.01)$ versus $93 \%(91 \%$ $95 \%$ ) in other adults (difference $P<0.001$ ).

Conclusions: There were increased ART initiations in all patient groups after implementation of World Health Organization 2013 guidelines. Retention of Option $\mathrm{B}+$ women was poorer than retention of other adults; younger women require attention because they are more likely to disengage from care.

Key Words: antiretroviral therapy, Option B+, CD4 threshold, decentralization, primary health care

(J Acquir Immune Defic Syndr 2017;74:508-516)

\section{INTRODUCTION}

World Health Organization (WHO) 2013 guidelines promoted expanded eligibility for antiretroviral therapy (ART) by raising the CD4 threshold for treatment initiation from 350 to 500 cells per cubic millimeter for HIV-infected individuals aged 5 years and older. ART was recommended for all children younger than 5 years. Option B (ART during pregnancy and breastfeeding) or Option $\mathrm{B}+$ (provision of ART for life for pregnant/breastfeeding women) for high fertility/high HIV prevalence countries was recommended over Option A (ART perinatally only) for prevention of mother-to-child transmission (PMTCT). ${ }^{1}$ The PROMISE trial subsequently confirmed superiority of Option B over Option A for reducing mother-to-child transmission (MTCT). ${ }^{2}$ Malawi was the first country to implement "Option B+" in September 2011. ${ }^{3}$ By 2015, most low-/middle-income countries were piloting, rolling out, or implementing Option $\mathrm{B}+{ }^{4}$ Advantages of Option B+ over previous PMTCT Option A and Option B (ART during pregnancy and breastfeeding) 
include: simplification of PMTCT program requirements (notably no requirement for a CD4 count result to be returned before ART initiation); extended protection from MTCT in future pregnancies from conception; prevention against sexual transmission to serodiscordant partners; benefit to the woman's health of earlier treatment and avoiding the risks of stopping and starting ART; and a simple message to communities that once ART is started, it is taken for life. ${ }^{5}$

Striking increases in pregnant women accessing ART have been seen in countries implementing Option $\mathrm{B}+$, although the first countries to implement Option $\mathrm{B}+$ tended to have low PMTCT coverage. ${ }^{6,7}$ Because Option $\mathrm{B}+$ is relatively new, there are few reports of outcomes from national programs. In the first national cohort of Option $\mathrm{B}+$, women in Malawi retention at 12 months, when most African women are still breastfeeding, was only $77 \% \%^{7}$; retention within more recent cohorts is similar, ${ }^{8}$ emphasizing the need for support measures for this population. At Elizabeth Glazer Pediatric AIDS Foundation sites in Uganda, early 6-month data suggest slightly higher retention. ${ }^{6}$

Zimbabwe adopted Option B+ after a National Stakeholder Consultation in $2013^{9}$ and began piloting in July 2013 with rollout in 2014. National guidelines issued at the end of 2013 also included recommendations to treat all HIV-infected children younger than 5 years and to treat older children and adults with WHO stage $1 / 2$ disease and CD4 count $<500 .{ }^{10}$ Before the introduction of Option B+, the PMTCT program (providing Option A) in Zimbabwe was functioning comparatively well (78\% ART coverage in pregnant women in $2013^{11}$ ), population HIV and CD4 testing levels were high, and ART coverage was also high at $77 \%$ in treatment-eligible adults in $2013^{12}$ ), although considerably lower in children at $46 \%{ }^{12}$ However, ART clients were frequently traveling long distances to access treatment, and ART provision in primary care (where available) was principally through outreach teams visiting from local hospitals. Implementation of Option $\mathrm{B}+$ necessitated integration of ART into all PMTCT settings where services are mostly delivered by nurses at the primary health facility level; for Zimbabwe, this required expanding the ART program from 1006 to 1560 facilities between 2013 and 2015. ${ }^{9}$

The Lablite Project ${ }^{13}$ worked with Ministries of Health in Malawi, Uganda, and Zimbabwe to evaluate rollout of ART at 4 nonresearch sites (each comprising a district hospital and 2-4 linked rural primary care facilities). In Zimbabwe, Lablite was present in Zvimba, a rural district west of Harare, with 270,000 inhabitants and estimated HIV prevalence of $14 \%$. The district includes 2 secondary care facilities and 31 primary care facilities. Here, we report on ART provision during 6 months before implementation of Option B+ (March 2014) and 12 months after implementation, in 1 of the district hospitals and 3 primary care facilities. The CD4 threshold for starting ART was raised from 350 to 500 cells per cubic millimeter in January 2014, 2 months earlier than implementation of Option $\mathrm{B}+$; but in practice, few initiations at higher $\mathrm{CD} 4 \mathrm{~s}$ occurred before Option $\mathrm{B}+$ was rolled out. Aims of this article were to describe the impact of Option $\mathrm{B}+$ provision and the increase in CD4 initiation threshold on ART provision and to quantify retention on ART, identifying predictors of high retention in care.

\section{METHODS}

The Lablite facilities were selected in consultation with Ministry of Health and Child Welfare (MOHCW). A "hub-spoke" approach was taken, with Banket District Hospital being the hub for 3 primary care facilities; before the Lablite project, 2 facilities (Nyabira, Mutorashanga) were benefiting from ART provision by Banket District Hospital outreach teams fortnightly and 1 facility (Zowa) had no ART provision. In collaboration with $\mathrm{MOHCW}$, health care workers were trained and mentored on site using national training materials, supplemented by a checklist for monitoring patients on ART and a handbook. Staff at Banket District Hospital were trained first and they mentored staff in the primary care facilities. The primary care facilities then received accreditation as ART-initiating and follow-up sites.

Routine individual patient-level data were collected regularly from ART registers, appointment registers, and facility patient cards for all patients newly registered on ART between September 2013 and February 2015 by a data capturer independent from clinic staff. Registrations include patients who are initiating ART and patients described as "transfers-in" who have started ART at another health care facility and are moving facility for follow-up on ART (during this study, often because of primary care facilities now providing full ART services, when they had not previously). Defaulters (no visit for 90 days) were cross-checked with clinic defaulter lists (lists that the nurses are required to keep within each clinic to identify defaulters for $\mathrm{MOHCW}$ reporting). Data were entered in bespoke database tools that mimicked the clinic paper-based tools.

Reasons for starting ART were coded for analysis in a hierarchical manner as follows: children younger than 15 years, adults with WHO stage $3 / 4$ disease (including tuberculosis), pregnant post-Option $\mathrm{B}+$, breastfeeding postOption $\mathrm{B}+$, CD $4<$ threshold. Pregnancy and breastfeeding were coded as reasons for initiation above CD4 for 2 reasons: (1) Option B+ women started ART immediately without waiting for CD4 results and (2) not all pregnant/breastfeeding women have CD4 results. Current outcome at last data collection (between March and June 2015) was used to estimate retention on ART at the end of follow-up (February 28, 2015), gaps in care before last data collection were ignored. Patients who transferred out to a new health facility were censored at the date of transfer.

Comparisons of initiation characteristics were planned between 6-month periods, pre-Option $\mathrm{B}+$, and $0-6$ and 7-12 months post-Option $\mathrm{B}+$. Retention in care was estimated separately for new ART initiations and patients who transferred in on ART. Comparisons of retention on ART were planned between facilities and for new ART initiations between Option $\mathrm{B}+$ women and other adults. Proportions were compared using $\chi^{2}$ tests. Rank sum tests were used to compare distributions of continuous variables. Retention in care at 6 and 12 months was estimated by the Kaplan-Meier survivor function. Hazard ratios (HRs) were estimated using Cox regression; HRs and $P$-values presented in the text to assess differences in retention by patient-level characteristics and facility are from univariable models (log-rank tests gave 
similar $P$-values, data not shown). Multivariable Cox models were selected by backward selection with significance level of 0.1 ; continuous variables were included as fractional polynomials. In patients who started ART for WHO stage 3/4 disease or CD $4<$ threshold, we fitted separate models for the risks of death and default, including all the predictors identified for the combined endpoint (cause-specific HRs are presented). Cumulative incidence of death and default were estimated using a competing risks framework. ${ }^{14}$ STATA version 13.1 was used.

\section{Role of the Funding Source}

The study was funded by Department for International Development, United Kingdom, who had no role in study design, collection, analysis and interpretation of data, the writing of the paper, or the decision to submit for publication. The corresponding author had full access to all the data.

\section{RESULTS}

Between September 2013 and February 2015, there were 2088 ART registrations across the 4 facilities, including $1686(81 \%)$ new ART initiations and 402 (19\%) patients already on ART who transferred in from an alternative health facility; 702 (42\%) new initiations and 277 (69\%) transfers-in were at the primary care facilities.

In the 3 facilities with established ART provision before Option $\mathrm{B}+$ implementation, there were 300 ART initiations in the 6 months before Option $\mathrm{B}+$ rollout compared to 869 and 463 initiations in the $0-6$ months and 6-12 months afterward, respectively, corresponding to 2.9- and 1.5-fold increases, with the largest in the first quarter (Table 1 and Fig. 1). Initiations in children younger than 15 years comprised $36(12 \%), 60(7 \%)$, and $43(9 \%)$ of ART initiations in the same 3 respective periods. Infants 2 years and younger were in the minority and were seen primarily at the hospital; 37 of $92(40 \%)$ children were 2 years and younger at the hospital compared with 3 of $47(6 \%)$ at primary care facilities $(P<0.001)$. Throughout follow-up, adult males initiating ART were older $(P<0.001)$ and had lower pre-ART CD4s than nonpregnant/breastfeeding females $(P<0.001)$ (Table 1). From March 2014, the median (interquartile range) pre-ART CD4 rose from $143(71-243)$ to $216(104-326)$ in men $(P<0.001)$ and from $192(89-263)$ to $279(161-396)$ in nonpregnant/breastfeeding women $(P<0.001)$. However, even between March 2014 and February 2015, 79 of 329 (24\%) men and 47 of 362 (13\%) nonpregnant/breastfeeding women had pre-ART CD $\leq 100$ cells per cubic millimeter. There were 3 ART initiations in men at the hospital where reason given for initiation was "male partner of an Option B+ female"; although all were WHO stage 1, CD4s at initiation were 66,313 and 470 cells per cubic millimeter, so they were eligible for treatment for their own health.

Over the 6 months before rollout of Option $\mathrm{B}+, 34$ (24\%) women initiating ART were pregnant/breastfeeding, including 3 early Option $\mathrm{B}+$ initiations at the hospital; excluding these 3 and 1 woman who was not WHO staged, 21 of $30(70 \%)$ had WHO stage $3 / 4$ disease. In contrast, in the first quarter after Option B+ rollout, initiations rose to 269 among pregnant/breastfeeding women (corresponding to a 16-fold increase) and then reduced to 82, 42, and 42 in the subsequent 3 quarters (a 6-fold increase over the first year after Option B+). After Option B+, 59 of 435 (13\%) pregnant/ breastfeeding women were WHO stage 3 at ART initiation and none had stage 4 disease. CD4 data were incomplete [only 190 of $376(51 \%)$ with WHO stage $1 / 2$ had a CD4 recorded]; $64(34 \%)$ stage $1 / 2$ women had $\leq 350$ cells per cubic millimeter and $47(25 \%)$ had $351-500$ cells per cubic millimeter, implying that overall, $43 \%$ of pregnant/breastfeeding women would have been eligible for treatment of their own health based on WHO 2010 disease progression criteria (WHO stage $3 / 4$ or CD4 $\leq 350$ cells per cubic millimeter) or $64 \%$ based on WHO 2013 criteria (WHO stage $3 / 4$ or CD4 $\leq 500$ cells per cubic millimeter). Median age at ART initiation in pregnant/breastfeeding women was similar pre- and post-Option B+ (Table 1); but the proportion of adolescents younger than 20 years increased from $1(3 \%)$ before Option $\mathrm{B}+$ to $25(7 \%)$ in the first 6 months and 13 $(15 \%)$ in the second 6 months post-Option $\mathrm{B}+$ (trend $P=0.008)$.

In Zowa, a primary care facility, where ART provision was started later and alongside Option $\mathrm{B}+$ rollout (from April 2014), there were 54 ART initiations, including 6 in children younger than 15 years (Fig. 1).

Median follow-up in individuals newly initiating ART was 8 (3-11) months. Retention on ART in 1140 adults who started ART for disease progression (all patients pre-Option $\mathrm{B}+$ implementation; pregnant/breastfeeding women with WHO stage 3/4 disease, and nonpregnant/breastfeeding patients post-Option $\mathrm{B}+$ implementation) was $95 \%(95 \%$ confidence interval: $93 \%$ to $96 \%$ ) at 6 months, including 12 deaths and 39 losses to follow-up (LTFU); 12-month retention was 93\% (91\%-95\%) (18 deaths, 40 LTFU). Figure $2 \mathrm{~A}$ shows the cumulative incidence of death and default across all sites. Retention varied between sites (Table 2; $P<$ $0.001)$; $12-$ month retention in the primary care facilities was $86 \%(80 \%-90 \%)$ compared with $98 \%(96 \%-99 \%)$ at the hospital. There was no evidence that retention-in-care was worse when ART was initiated after Option B + had rolled out compared with before Option $\mathrm{B}+$ (Table 2). Retention was higher in patients with higher CD4 and lower in pregnant/ breastfeeding patients (pre-Option $\mathrm{B}+$ or also WHO stage 3/4); when we looked at the risks of death and LTFU separately, the CD4 effect was predominantly because of an increased risk of death in patients with low CD4, whereas pregnancy/breastfeeding predicted higher LTFU but not higher mortality (Table 3 ). Among WHO stage $1 / 2$ patients, those with CD4 $>350$ had similar retention to those with CD4 $\leq 350$ [HR: $1.11(0.63-1.94)]$

Retention on ART in 386 women who newly started ART for Option B+ was $85 \%(81 \%-88 \%)$ at 6 months (1 death, 53 LTFU) and $79 \%(69 \%-87 \%)$ at 12 months (3 deaths, 55 LTFU); this was significantly lower than retention in the adults starting ART for WHO $3 / 4$ or CD $<<$ threshold [across all periods: HR 3.09 (2.15-4.46), $P<0.001$; restricted to March 2014 onward: HR 4.00 (2.59-6.09), $P<0.001]$. Retention differed markedly by age at initiation, 
TABLE 1. Characteristics at ART Initiation in a District Hospital and 2 Primary Care Facilities With Established ART Provision Before Option B+ Implementation*

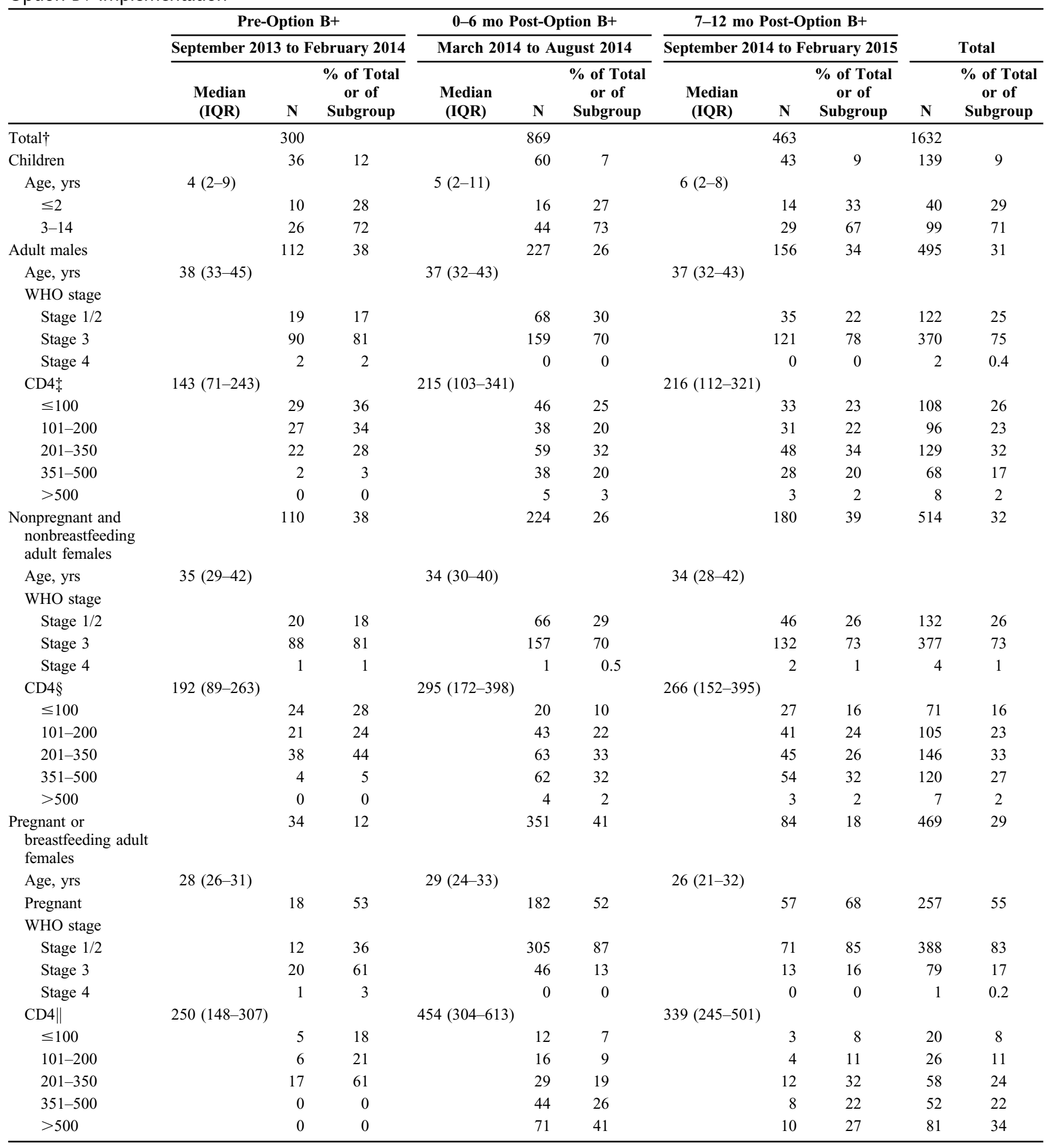

*Zowa primary care health facility is not included as there was no ART provision before Option B+, hence its inclusion post-Option B+ could distort comparisons. $\dagger 15$ individuals with missing age excluded from sub-groups below.

†CD4 at initiation was available in $80(71 \%), 186(82 \%), 143(92 \%)$ males by period.

§CD4 at initiation was available in $87(79 \%), 192(86 \%), 167(93 \%)$ nonpregnant and nonbreastfeeding females by period.

\|CD4 at initiation was available in $28(82 \%), 172(49 \%), 37(44 \%)$ pregnant or breastfeeding females by period.

$\mathrm{IQR}$, interquartile range. 


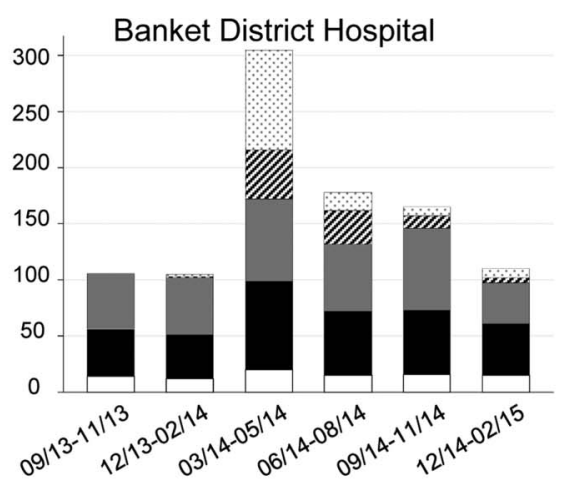

A

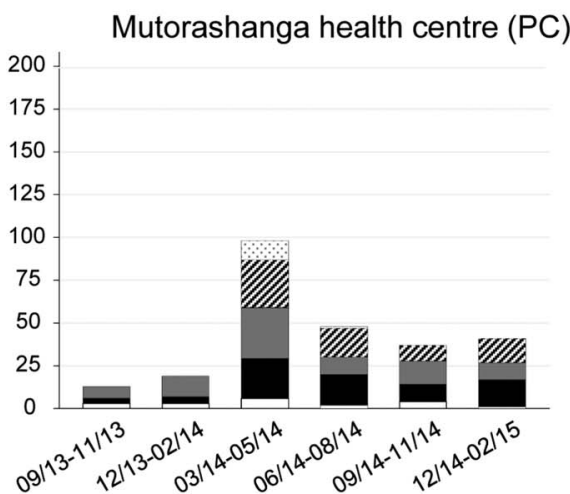

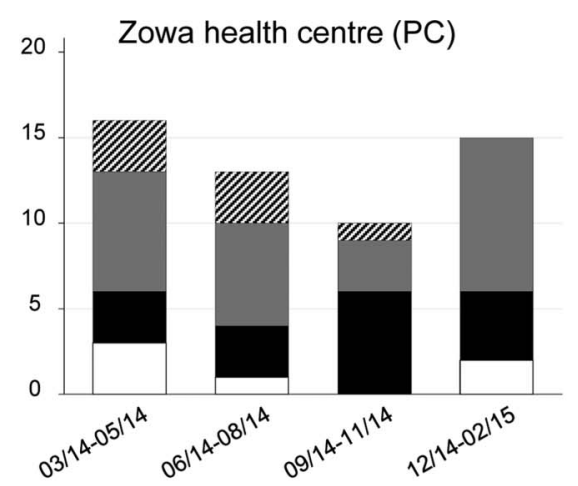

B

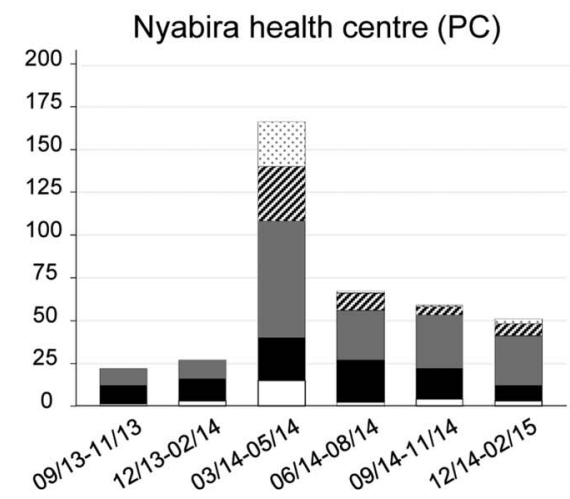

FIGURE 1. A-D, Number of new ART initiations by patient status at ART initiation, period and health facility (PC, primary care facility).
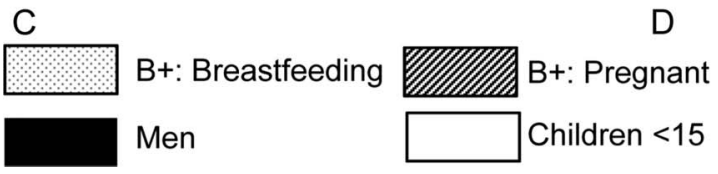

Non B+ women

being lower in younger women (Table 2 and Fig. 2B). Retention did not differ by pre-ART CD4 (Table 2). Although 32 women were LTFU by 1 month, 20 of 32 attended 1 postART initiation visit (median 14 days after starting ART). Importantly, in the subgroup of women who started ART during pregnancy/breastfeeding with WHO stage 3/4 or CD4 $\leq 350$, there was no evidence for worse retention after Option B+ implementation than before [HR: 0.78 (0.28-2.16)].

Among 145 children younger than 15 years at ART initiation, there were $3(2 \%)$ deaths, 16 (11\%) LTFU, and 10 (7\%) transfers-out. The 3 children who died were all seen at the hospital; two 3-year-olds died very soon after starting
ART (13 days; 45 days) and one 2-year-old died 12 months after initiation. At 6 months, retention on ART was $87 \%$ $(80 \%-92 \%)$ and at 12 months it was $85 \%(77 \%-91 \%)$. We found no evidence that retention at 12 months in the primary care facilities $[94 \%(83 \%-98 \%)]$ was worse than at the hospital $[79 \%(67 \%-88 \%)]$.

Retention on ART in the 402 individuals who transferred into one of the facilities already on ART was 95\% (92\%-97\%) 6 months after transfer (0 death, 18 LTFU) and $94 \%(90 \%-96 \%)$ (0 death, 20 LTFU) at 12 months. Over half the individuals LTFU $(11 / 20)$ did not return after their first registration visit.
FIGURE 2. A and B, Cumulative incidence of death or default (LTFU) by time since ART initiation. *Option $\mathrm{B}+$ women defined as women starting ART after Option B+ provision, who were $\mathrm{WHO}$ stage 1 or 2 and pregnant or breastfeeding.
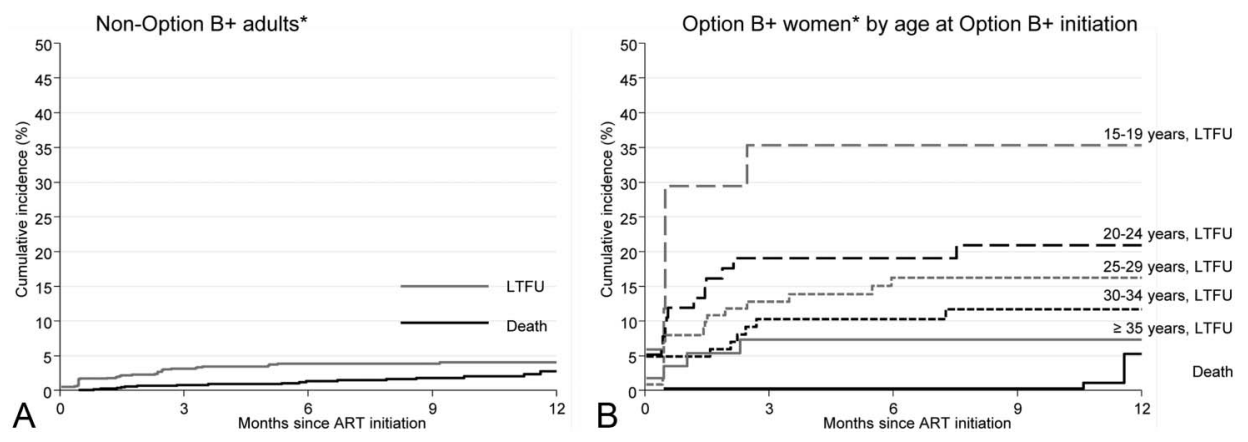
TABLE 2. Predictors of Death and Loss to Follow-up From Care in Adults Newly Starting ART

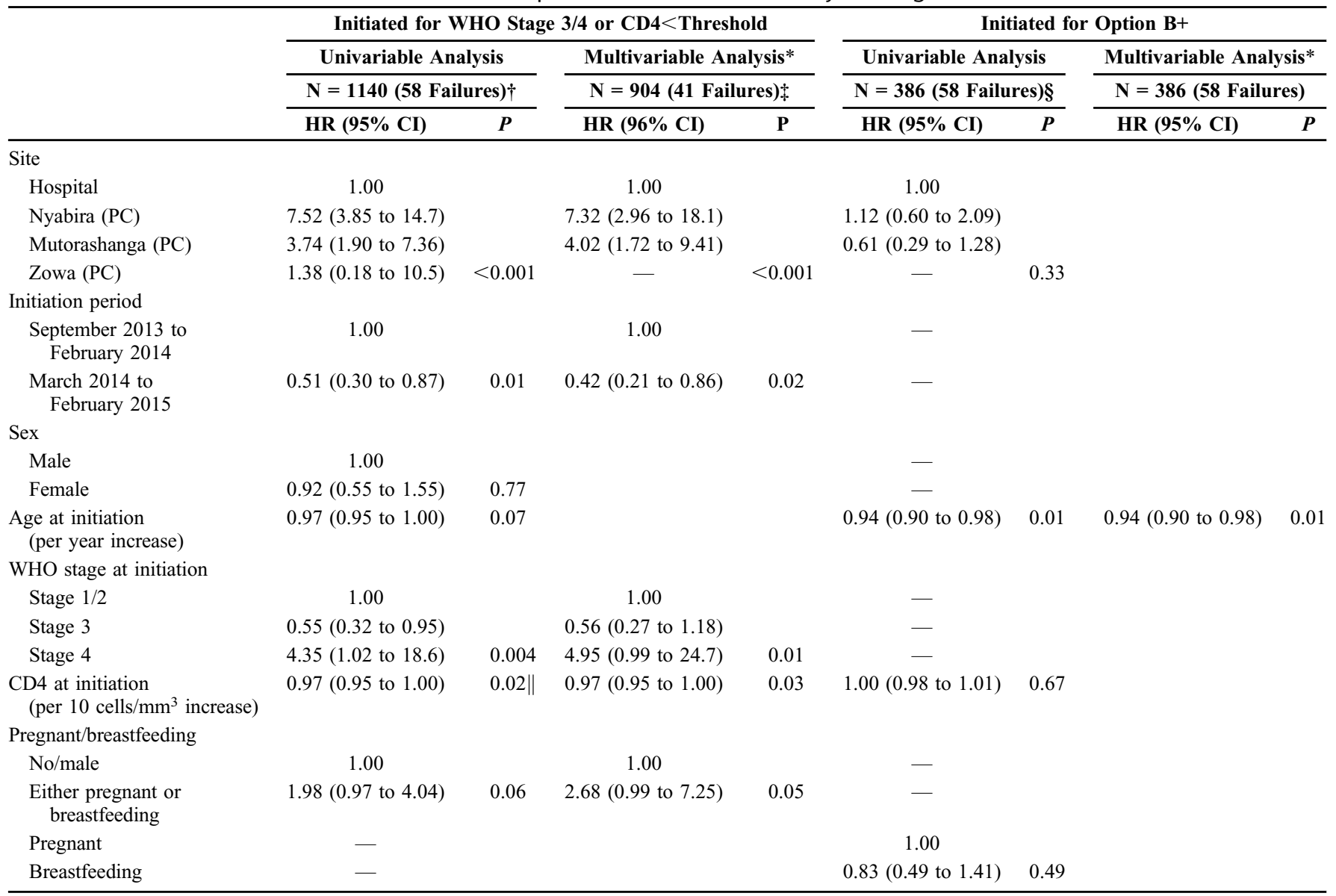

*All variables presented in the univariable analysis were considered for inclusion in the multivariable model. Continuous variables (CD4 and age) were included as fractional polynomials for model selection, which was based on backward elimination $(P<0.10)$.

$\dagger$ Because of missing data, CD4 model $(\mathrm{N}=944$, failures $=41)$; WHO stage model $(\mathrm{N}=1137$, failures $=58)$.

$\$$ Patients with missing CD4 or WHO stage or at Zowa (no pre-Option B+ data) were dropped in the final model.

§Because of missing data, CD4 model $(\mathrm{N}=193$, failures $=28)$; because of no failures at Zowa, site model $(\mathrm{N}=379$, failures $=58)$

$\| \mathrm{CD} 4$ in the univariable model was better represented by $\mathrm{CD} 4{ }^{-0.5}$ but the linear term is shown here for comparability with the multivariable model and for ease of interpretation. $\mathrm{CI}$, confidence interval; PC, primary care facility.

\section{DISCUSSION}

The last 5 years have seen rapid changes in both PMTCT guidance and practice and CD4 threshold recommendations for ART initiation. The Lablite project $^{13}$ was working with Ministries of Health in Malawi, Zimbabwe, and Uganda between 2011 and 2015 and collating routine data on ART rollout in nonresearch facilities in rural areas. In Zimbabwe, this provided an important opportunity to compare ART initiations and retention on ART before and after introduction of Option B + and the increase in CD4 threshold.

We found that after Option $\mathrm{B}+$ implementation and the increased CD4 threshold, greater numbers of all patients started ART, including children. Patients who started ART at CD4 $>350$ or for Option B+ (with high CD4) inevitably did so as a result of guideline changes; however, increases in other patient groups may have occurred anyway. Devolvement of ART care to the primary care staff with support from $\mathrm{MOHCW}$ allowed management of increasing patient num- bers. However, drug supply remains a concern; although no stock-outs of ART were reported, there were anecdotal reports of patients having to return to clinic in between scheduled visits to collect ART because of low supplies, and stock-outs of cotrimoxazole did occur.

Overall mortality was low and retention in care was extremely good in patients starting ART for disease progression ( $93 \%$ at 12 months), better than reported for the ART program in Zimbabwe between 2007 and $2010^{15}$ and in other settings. ${ }^{16}$ Retention was higher at the hospital than in the primary care facilities, possibly because of the Lablite model of training whereby hub staff received additional training to mentor staff at primary care facilities. Encouragingly, we found no evidence that introduction of Option B+ had a detrimental effect on retention of patients starting ART for disease progression. Retention on ART was poorer in Option $\mathrm{B}+$ women than in other adults starting ART, as found in Malawi, ${ }^{17}$ but similar to retention reported in other rural 
TABLE 3. Predictors of Death and Loss to Follow-up From Care in Adults Newly Starting ART for WHO Stage $3 / 4$ or CD4 $<$ Threshold

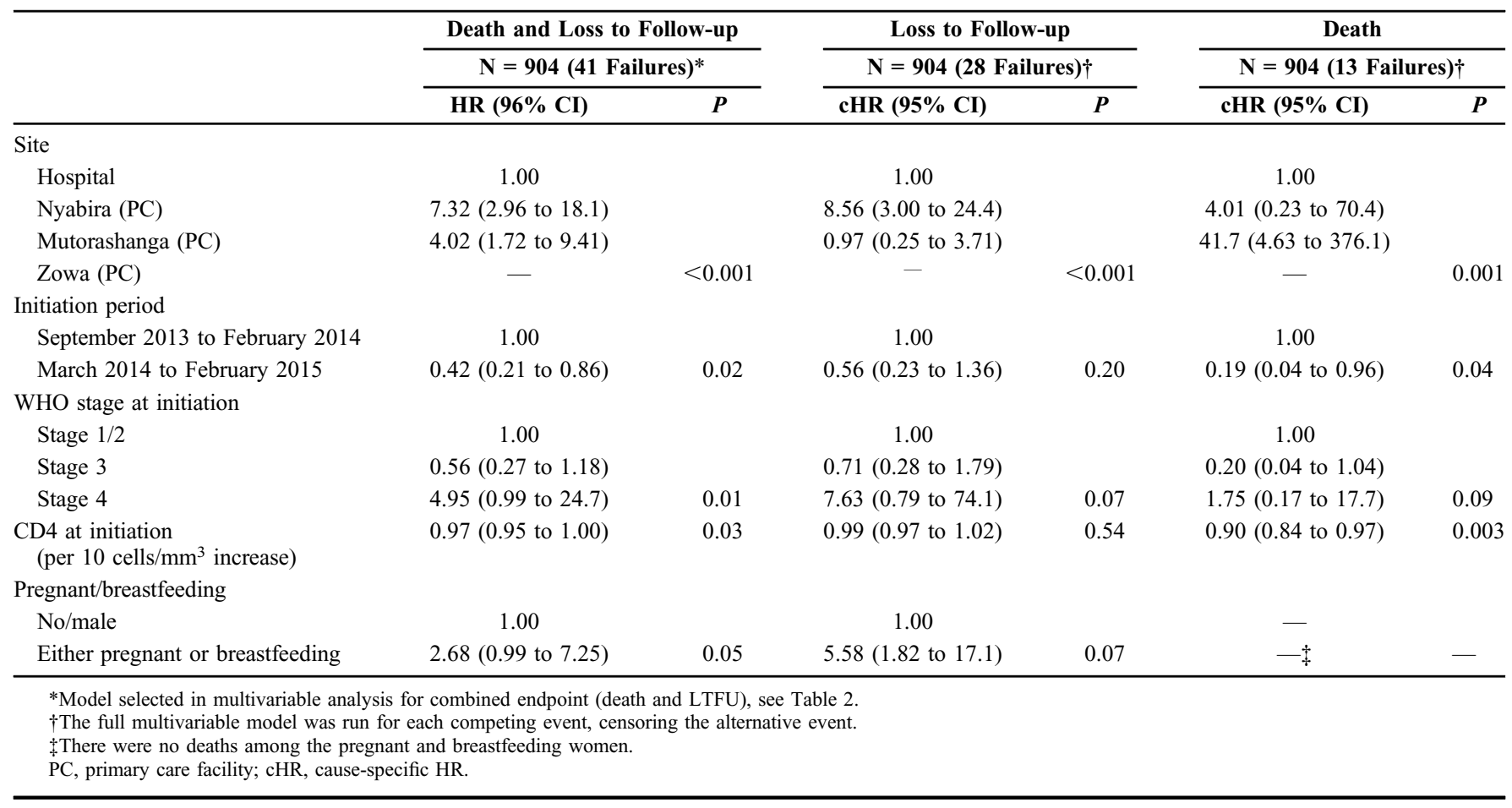

districts of Zimbabwe (83\%). ${ }^{18}$ At all 4 Lablite facilities, women were asked to start ART immediately; for many, HIV is a new diagnosis after routine testing during antenatal care, and qualitative work suggests that immediate treatment may be difficult because of the limited time available to digest a positive HIV diagnosis and understand and form decisions around the uptake of a new lifelong treatment. ${ }^{17,19}$

CD4 counts were obtained for $85 \%$ of men and nonpregnant/breastfeeding women before ART with no drop in testing after Option B+ implementation or increase in CD4 threshold. Numbers of men and nonpregnant/breastfeeding women starting ART with CD4 $>350$ were encouraging, suggesting these individuals can be identified, although we could not distinguish individuals newly diagnosed from those already in pre-ART HIV care. Despite this, a significant proportion of individuals still start ART with low CD4s, particularly men, who are more likely to present later than women. ${ }^{20,21}$ Around half Option B+ women had a pre-ART CD4 recorded; facilities were asked to continue providing CD4s (unlike in Malawi where CD4s are not offered to pregnant/breastfeeding women ${ }^{13}$ ); it is unclear whether remaining women had no CD4 measured or whether it was not recorded. Assuming recorded $\mathrm{CD} 4 \mathrm{~s}$ were representative in Option $\mathrm{B}+$ women, importantly $>40 \%$ pregnant/breastfeeding women needed ART for their own disease (either WHO stage $3 / 4$ or CD4 $\leq 350$ ), consistent with other studies. ${ }^{22}$ It is important that these women are retained on ART for their own health and to prevent MTCT; health care workers may need focused training to support retention of Option $\mathrm{B}+$ women with low CD4 counts.
Among Option B+ women, almost all attrition was recorded as LTFU; unrecorded deaths are unlikely to be a major contributor but we cannot distinguish between women who have "silently" transferred to other health facilities and those who have dropped out of care completely. Age was a clear factor in predicting LTFU, with younger women more likely to be lost. This corroborates findings in Malawi ${ }^{23,24}$ and Zimbabwe. ${ }^{18}$ Targeted support measures are needed for the increasing numbers of HIV-infected adolescent and young pregnant women starting ART. We were only able to look at retention on ART to 12 months, and it is important to recognize that losses in this period, most of which were very early, are likely to have been during pregnancy or breastfeeding. In Malawi, it is estimated that half of those lost in the first 6 months may never have started ART. ${ }^{8}$ In Zimbabwe, patients are asked to return to clinic 2 weeks after starting ART, which is earlier than elsewhere (including Malawi). We found that around 2/3 of women who were lost by 1 month had returned for their 2 -week visit; this raises concerns that they took ART for only a short time and may now have developed resistance as the half-life of efavirenz may be much longer than other drugs. Data from Malawi suggest that the risk of LTFU is much lower after the first year ${ }^{24}$; however, it will be some time before comparable data are available for Zimbabwe.

In Zimbabwe since Option B+ rollout, policy has been to encourage male partners to attend antenatal care with pregnant women and to provide them with ART, irrespective of their own CD4 count. However, this seems to have happened very rarely (only 3 men). Initiatives to engage 
men are critical as lack of male involvement and the complex dynamics between the women and their partners including concerns about stigma are cited by health care workers as a common reason for nonretention among women on Option B+. ${ }^{25,26}$

Historically, HIV-infected children have been seen in secondary and tertiary health care facilities in Zimbabwe, ${ }^{27}$ so it was encouraging to see ART initiations in under 15's at all facilities, including the new primary care facility (where ART provision was introduced alongside Option $\mathrm{B}+$ ). However, similar to other studies, ${ }^{28}$ children younger than 24 months were treated almost exclusively at the hospital, suggesting possible reluctance to test and treat infants in primary care. Retention on ART was also somewhat lower in children than in adults starting ART outside of Option B+. Previous studies of retention in care of $\mathrm{HIV}$-exposed and HIV-infected children have identified a range of possible risk factors, including characteristics of the carer for mortality and $\mathrm{LTFU}^{29-31}$; further research is needed to develop strategies to mitigate losses.

After the START and TEMPRANO trials, ${ }^{32,33}$ WHO released new guidelines recommending that all HIV-infected individuals should start treatment as soon as possible after a positive diagnosis. ${ }^{34}$ It is unclear how quickly this recommendation will be taken up in sub-Saharan Africa. Our early data on uptake of treatment in individuals with CD4 $>350$ after the rise in threshold suggest that there will be demand. If adopted, lessons may be learned from Option B+ rollout; immediate treatment after provider-initiated testing in "well" patients may not be dissimilar. In particular, adopting treatment for all may facilitate retention of pregnant/breastfeeding women by reducing the current disparity in access to ART between women and their partners.

Strengths of the Lablite project include the study of rural $\mathrm{MOHCW}$ facilities with little/no research experience and use of routine data collected from paper records. This contrasts with much of the existing Option $\mathrm{B}+$ operations research, which primarily focuses on larger health facilities with electronic data capture. Additionally, we have data before and after the introduction of Option $\mathrm{B}+$ and changes in WHO CD4 threshold guidelines and across all patient groups. The primary weaknesses include lack of information on the underlying causes of patients LTFU (common to most studies) and use of a retrospective definition of LTFU (often used in analysis of routinely collected data where visit data may be incomplete). Our study is limited to 4 facilities and it remains important to collate further data to determine how generalizable our findings are.

\section{ACKNOWLEDGMENTS}

The Lablite Team comprises: Dignitas International, Zomba, Malawi: F.C., A.K.C., L. Chiwaula, M.J.N., J. J. van Oosterhout, G.Mateyu, M. Willie; MRC/UVRI Uganda Research Unit on AIDS, Entebbe, Uganda: F. Mirimo, S. Kiwuwa, J.S.; Joint Clinical Research Centre, Kampala, Uganda: G.A., C.K., Harriet Namata; University of Zimbabwe, Harare, Zimbabwe: J.H., T.M., M.M., A.R., M.N.; Infectious
Diseases Institute, Makerere University, Mulago, Uganda: S. Kaggwa, E.K., I. Mambule. School of Population Health, University of Queensland, Australia: C.F.G.; Centre for Health Economics, University of York, UK: P. Revill; MRC Clinical Trials Unit at UCL, London, United Kingdom: D.F., D.M.G., C.G., S. Hoskins, S.J., A. South, M. Thomason. Independent Chair of the Project Management Group: I. Weller. The Lablite Zimbabwe team acknowledges the guiding role of the Central Ministry of Health and Child Care including Provincial Medical Directorates, City of Harare Directorate of Health Services, District Health Executive Teams, and all facility staff involved. The authors also extend their gratitude to the former DART staff at the University of Zimbabwe Clinical Research Centre.

\section{REFERENCES}

1. World Health Organization 2013. Consolidated Guidelines on the Use of Antiretroviral Drugs for Treating and Preventing HIV Infection. Available at: http://www.who.int/hiv/pub/guidelines/arv2013/download/en/ index.html. Accessed October 21, 2015.

2. Fowler MG, Qin M, Fiscus SA, et al. PROMISE: efficacy and saftey of 2 strategies to prevent perinatal HIV transmission. CROI 2015. Available at: http://www.croiconference.org/sessions/promise-efficacy-and-safety2-strategies-prevent-perinatal-hiv-transmission. Accessed January 12, 2016. 2015.

3. Clinical Management of HIV in Children and Adults. Malawi Integrated Guidelines: Malawi: Ministry of Health; 2011.

4. World Health Organization 2015. Global Health Sector Response to HIV, 2000-2015: Focus on Innovations in Africa: Progress Report. Available at: http://apps.who.int/iris/bitstream/10665/198065/1/9789241509824_eng. pdf?ua=1. Accessed January 12, 2016.

5. Schouten EJ, Jahn A, Midiani D, et al. Prevention of mother-to-child transmission of HIV and the health-related Millennium Development Goals: time for a public health approach. Lancet. 2011;378:282-284.

6. Kieffer MP, Mattingly M, Giphart A, et al. Lessons learned from early implementation of option $\mathrm{B}+$ : the Elizabeth Glaser Pediatric AIDS Foundation experience in 11 African countries. J Acquir Immune Defic Syndr. 2014;67(suppl 4):S188-S194.

7. Impact of an innovative approach to prevent mother-to-child transmission of HIV-Malawi, July 2011-September 2012. MMWR Morb Mortal Wkly Rep. 2013;62:148-151. See ref. https://www.cdc.gov/ $\mathrm{mmwr} / \mathrm{preview} / \mathrm{mmwrhtml} / \mathrm{mm} 6208 \mathrm{a} 3 . \mathrm{htm}$.

8. Integrated HIV Program Report January-March 2015. Government of Malawi: Malawi: Ministry of Health; 2015.

9. An Operational Plan for the Nationwide Transition to Option $\mathrm{B}+$ in Zimbabwe. Zimbabwe: Ministry of Health and Child Care; 2013.

10. National Medicine and Therapeutics Policy Advisory Committee (NMTPAC) and The AIDS and TB Directorate. Guidelines for Antiretroviral Therapy for the Prevention and Treatment of HIV in Zimbabwe: Zimbabwe: Ministry of Health and Child Care; 2013.

11. World Health Organization 2014. Global Update on the Health Sector Response to HIV, 2014. Available at: http://apps.who.int/iris/bitstream/ 10665/128494/1/9789241507585_eng.pdf?ua=1. Accessed January 5, 2016.

12. National AIDS Council of Zimbabwe. 2015. Global AIDS Response Country Progress Report Zimbabwe 2014. Available at: http://www.nac. org.zw/sites/default/files/Zimbabwe\%20Country\%20report2014_0.pdf Accessed January 12, 2016.

13. The Lablite Project. Available at: http://lablite.org/Accessed January 5, 2016.

14. Tai BC, White IR, Gebski V, et al. On the issue of "multiple" first failures in competing risks analysis. Stat Med. 2002;21:2243-2255.

15. Mutasa-Apollo T, Shiraishi RW, Takarinda KC, et al. Patient retention, clinical outcomes and attrition-associated factors of HIV-infected patients enrolled in Zimbabwe's National Antiretroviral Therapy Programme, 2007-2010. PLoS One. 2014;9:e86305.

16. Tassie JM, Baijal P, Vitoria MA, et al. Trends in retention on antiretroviral therapy in national programs in low-income and middle-income countries. J Acquir Immune Defic Syndr. 2010;54:437-441. 
17. Tenthani L, Haas AD, Tweya $\mathrm{H}$, et al. Retention in care under universal antiretroviral therapy for HIV-infected pregnant and breastfeeding women ("Option B+") in Malawi. AIDS. 2014;28:589-598.

18. Dzangare J, Takarinda KC, Harries AD, et al. HIV testing uptake and retention in care of HIV-infected pregnant and breastfeeding women initiated on "Option B+" in rural Zimbabwe. Trop Med Int Health. 2015; 21:202-209.

19. Cataldo F. Exploring women and health care workers experiences in the context of PMTCT Option B Plus in Malawi. Oral presentation at ICASA; December 7-11, 2013; Capetown. Abstract 2429707.

20. Cornell M, Schomaker M, Garone DB, et al. Gender differences in survival among adult patients starting antiretroviral therapy in South Africa: a multicentre cohort study. PLoS Med. 2012;9:e1001304.

21. Takarinda KC, Harries AD, Shiraishi RW, et al. Gender-related differences in outcomes and attrition on antiretroviral treatment among an HIV-infected patient cohort in Zimbabwe: 2007-2010. Int J Infect Dis. 2015;30:98-105.

22. Carter RJ, Dugan K, El-Sadr WM, et al. CD4+ cell count testing more effective than HIV disease clinical staging in identifying pregnant and postpartum women eligible for antiretroviral therapy in resource-limited settings. J Acquir Immune Defic Syndr. 2010;55:404-410.

23. Tweya $\mathrm{H}$, Gugsa $\mathrm{S}$, Hosseinipour $\mathrm{M}$, et al. Understanding factors, outcomes and reasons for loss to follow-up among women in Option B+ PMTCT programme in Lilongwe, Malawi. Trop Med Int Health. 2014; 19:1360-1366.

24. Haas AD, Tenthani L, Msukwa MT, et al. Retention in care during the first 3 years of antiretroviral therapy for women in Malawi's option B+ programme: an observational cohort study. Lancet HIV. 2016;3:e175-182.

25. Mills EJ, Beyrer C, Birungi J, et al. Engaging men in prevention and care for HIV/AIDS in Africa. PLoS Med. 2012;9:e1001167.
26. Sonke Gender Justice Project 2015. Available at: http://www. genderjustice.org.za/publication/unaids-2015-discussion-paper/. Accessed March 5, 2016.

27. AIDS and TB Unit. The National OI/ART Programme Annual Report, 2009. Zimbabwe: Ministry of Health and Child Welfare; 2009.

28. Fayorsey RN, Saito S, Carter RJ, et al. Decentralization of pediatric HIV care and treatment in five sub-Saharan African countries. $J$ Acquir Immune Defic Syndr. 2013;62:e124-130.

29. Tene G, Lahuerta M, Teasdale C, et al. High retention among HIVinfected children in Rwanda during scale-up and decentralization of HIV care and treatment programs, 2004 to 2010. Pediatr Infect Dis J. 2013; 32:e341-347.

30. Sengayi M, Dwane N, Marinda E, et al. Predictors of loss to follow-up among children in the first and second years of antiretroviral treatment in Johannesburg, South Africa. Glob Health Action. 2013;6:19248.

31. Bigna JJ, Noubiap JJ, Plottel CS, et al. Factors associated with nonadherence to scheduled medical follow-up appointments among Cameroonian children requiring HIV care: a case-control analysis of the usual-care group in the MORE CARE trial. Infect Dis Poverty. 2014;3:44.

32. Group ISS; Lundgren JD, Babiker AG, et al. Initiation of antiretroviral therapy in early asymptomatic HIV infection. $N$ Engl J Med. 2015;373: 795-807.

33. Group TAS, Danel C, Moh R, et al. A trial of early antiretrovirals and isoniazid preventive therapy in Africa. N Engl J Med. 2015;373:808822.

34. World Health Orgnaization September 2015. Guideline on when to Start Antiretroviral Therapy and on Pre-exposure Prophylaxis for HIV. Available at: http://apps.who.int/iris/bitstream/10665/186275/1/9789241509565_eng. pdf?ua $=1$. Accessed January 12, 2016. 